\title{
Bemerkungen zum Problem der Existenz mathematischer Gegenstände.
}

\author{
Von Fritz Medicus in Zürich.
}

Es mag dem Verfasser eriaubt sein, seinen Ausführungen ein paar Worte persönlicher Art vorauszuschicken.

Vor einer Reihe von Jahren - es war noch im vorigen Jahrhundert - sprach mein Lehrer Riehl einmal mit mir über die Eigenart der mathematischen Existenz und meinte: „Das wäre ein Thema für Sie!* Ich hatte nicht lange vorher in meiner Dissertation die Netageometrie behandelt und wohl anch sonst gelegentlich mathematische Interessen verraten. Doch hahen bald Aufgaben anderen Charakters meine Fähigkeiten zur Bereichernng der Literatur völlig in Anspruch genommen, sodass in meinen Publikationen von Mathematik nichts mehr zu merken war. Dass ich gleichwohl jenen Forschlag Riehls errogen und - weun auch spät - befolgt habe, beweisen die vorliegenden Blättel. Ich freue mich, sie dem hochverehrten Manne widmen zu dürfen - und zwar in dieser Zeitschrift, d. h. nnter dem Zeichen des grossen Begründers der neuesten Philosophie, dessen Verständnis mir zuerst und in entscheidender Weise von ihm erschlossen worden ist.

„Urteile sind nicht lediglich begriffliche Vereinigungen von Torstellungen, sie gelten anch, sie bedeuten etrras vom Gegenstande, der durch sie beurteilt wird, mit einem Worte: sie sind Erkenntnisse. Die Beziehung auf ein Objekt ist dem Urteil Fesentlich ... Nicht jeder Satz ist ein Urteil, sondern nur derjenige ist es, bei welchem Wabrheit oder Falschbeit stattfinden kann, bemerkte schon Aristoteles, and es ist ein Fehler der formalistischen Logik, sich diese Bemerkung des Aristoteles nicht zunntze gemacht za haben. Wir können Begriffe willkürlich und zugleich in formal richtiger Weise verbinden, ohne durch diese Verbindung etwas za erkeunen oder auch nur erkennen za wollen, 
d. i. ohne in Wahrheit $\mathrm{zu}$ urteilen. $\mathrm{Zu}$ Urteilen werden formal richtige Sätze erst dadurch, dass wir zugleich ihre Gültigkeit von Objekten, oder Verhältnissen von:Objekten mitbehaupten" (Alois Riehl, Der philosophische Kritizismus, 2. Aufl., I, 417 f.). Es versteht sich, dass die Urteile der Mathematik keine Ausnahme machen: auch sie haben ihre Objekte, anch sie gelten gegenständlich, auch sie reden von Existierendem. Freilich - der Begriff der Existenz ist mehrdeutig. Existenzialaussagen können logisch sehr verschiedenen Charakter haben. „Es gibt Irrationalzahlen:", „Es gibt nur eine Art Schall, dagegen viele Arten Licht: natürliches, geradlinig polarisiertes, kreisförmig polarisiertes, elliptisch polarisiertes usw.", „Es gibt Jupitermonde", "Es hat einen Kirchenstaat gegeben", "Es wird Regen geben", „Es gibt keine Gespenster", "Es ist ungewiss, ob es einen Abraham aus Ur in Chaldäa gegeben hat": - diese Ürteile behaupten oder verneinen oder bezweifeln Existenz. Aber die Irrationalzahlen existieren in anderem Sinne als die Naturobjekte oder die historischen Gebilde. Und zwar müssen nicht nur Artén, sondern Grade der Existenz unterschieden werden. Schon jedes impersonale Urteil sagt - so gewiss es ein Urteil ist - Existenz aus : aber nicht eine Existenz des Seins, sondern eine solche des blossen Geschehens. „Es blitzt": gemeint ist hier weder bloss die Anschauung des Blitzes, noch enthält das Urteil schon etwas von begrifflicher Wesenserkenntnis des Vorganges; sondern der Inhalt der Aussage ist die Einordnung einer auschaulich-aktuellen Vorstellung in den $\mathrm{Zu}$ sammenhang des Wirklichen - aber nur des gegenwärtig Wirklichen. Wollte man das Urteil "Es blitzt" in dieser Form ohne weiteres festhalten, so müsste seine Wahrheit alsbald verschwinden: es kann wahr bleiben nur dadurch, dass es eingestellt wird in einen übergreifenden Urteilszusammenhang, in dem die flüchtige Existenz des blossen Geschehens befestigt ist in einem dauernd Existierenden.

Die Einsicht, dass es mannigfach abgestufte Grade der Existenz gibt, ist wichtig, um zu verstehen, wie in jedem Urteil irgendein Grad von Existenz unausgesprochen vorausgesetzt ist. Im Existenzialurteil handelt es sich niemals um Existenz im kontradiktorischen Gegensatz zur Nichtexistenz, sondern es handelt sich um einen bestimmten Grad von Existenz, der sich über einen vorausgesetzten niedrigeren Grad erhebt; und das negative Existenzialurteil spricht dem Urteilssubjekt niemals jed we'de Existenz 
$a b$, es bestreitet ihm lediglich einen bestimmten Existenzgrad. Wenn ich sage: „Es gibt keinen vierdimensionalen Raum“, so rede ich von einem Gebilde, das als Produkt mathematischer Konstruktion allerdings Existenz hat: es hat diejenige Art und denjenigen Grad von Existenz, der den Gebilden der Mathematik eigen ist; mein negatives Existenzialurteil will nur sagen, dass die Existenz dieses Gebildes nicht über die Existenzsphäre hinausreicht, in die es von selbst hineingehört, un überhaupt Subjekt dieses Urteils sein zu können. Das schlechthin in keiner Beziehung Existierende kann vie Träger eines Prädikats sein. ${ }^{1}$ )

Derselbe Existenzgrad nun, der in dem letzten Beispiel als selbstverständlich vorausgesetzt war, und ohne dessen Voraussetzung das Urteil gar nicht verstanden werden könnte, kann in einem anderen Urteil gerade der prädizierte Inhalt selbst sein. Einem mathematisch sehr harmlosen Gemüt kann man etwa sagen: "In der Mathematik gibt es auch den vierdimensionalen Raum". Derselbe Existenzgrad, der in dem ersten Urteil als nichtig angesprochen wurde, wird hier als etwas Positives behandelt. Der Begriff Existenz ist, wie man sieht, relativ.

Und wenn ich behaupte: "Es gibt den dreidimensionalen ebenen Raum, der dreidimensionale ebene Raum hat Realität", so iśt die Meinung: dĩe Existenz dieses Gebildes mathematischer Konstruktion erschöpft sich nicht in der Sphäre der Mathematik, sondern sie reicht darüber hinaus; der dreidimensionale ebene Ranm fasst nicht nur geometrische Konstruktionen in sich, sondern er umfasst auch Realitäten von anderer und zwar höherer Art. Sage ich: „Es gibt in der Natur keinen vollkommenen Kreis“, so enthält mein Urteil doch zugleich die Behauptung, dass der vollkommene Kreis allerdings existiert, nur eben in einer niedrigeren Existenzsphäre als der der Natur; ohne solche Voraussetzung wäre das Urteil sinnlos. Sein genauer Sinn ist der, dass dem in einer niedrigeren Existenzsphäre Existierenden kein Gegenstand der empirischen Anschauung vollkommen entspricht. - In jedem Existenzialurteil werden in solcher Weise zwei logisch verschiedene Existenzweisen gedacht. Wenn ich behaupte: „Es ist zweifelhaft, ob Abraham aus Ur in Chaldäa wirklich gelebt hat", so wäre es absurd, die Frage auf Existenz und absolute Nicht-Existenz abzu-

1) Vgl. Benedetto Croce, Logica come scienza del concetto puro, 2nda ed., Bari 1909, p. $116 \mathrm{f}$. 
stellen. Die absolnte Nicht-Existenz ist kein Objekt für die logische Funktion. Dass jener Patriarch in den Zusammenhängen der alttestamentlichen Geschichtsberichte existiert, und dass er von da aus auch bedeutsam auf die historische Realität eingewirkt hat, steht ausser jedem Zweifel, und die Bejahung dieser Existenz macht das angegebene Urteil ersț möglich. Problematisch ist lediglich die Eingliederung jenes Abraham als einer historischen Persönlichkeit in den Zusammenhang des geschichtlich Wirklichen. Wenn ein antimodernistischer Historiker die These ausspricht, der Patriarch Abraham habe wirklich existiert, so meint er dies durchaus nicht im Gegensatz zu absoluter Nicht-Existenz. Irgendwelche Art von Existenz ist in jedem Urteil vorausgesetzt, und diese vorausgesetzte Existenz, ohne die das Urteilssubjekt selbst nicht als solches möglich wäre, kann niemals Gegenstand der Aussage sein. Gegenstand der Aussage, d. h. Prädikat ist im Existenzialurteil nie etwas anderes als die Zugehörigkeit des Subjekts zu einem Existenzzusammenhang von höherem Grade als dem als selbstverständlich vorauszusetzenden.

Man darf sich nicht von der sprachlichen Form verführeu lassen, die den Anschein erwecken könnte, als handle es sich im Existenzialurteil um ein is oliertes Objekt, dem Existenz zu- oder abgesprochen werden sollte: in Wahrheit ist stets das Verhältnis des Objektes zu einem Wirklichkeitszusammenhang in Frage. Und nur wenn man dies festhält, versteht man, dass die Existenz wirklich Prädikat sein kann. Davon also ist gewiss keine Rede, dass z. B. dieses Ştück Papier neben den Prädikaten "weiss“, "glatt" usw. auch das Merkmal „wirklich" hätte; mit solchen Wähne hat Kant aufgeräumt. Wo die Merkmale "weiss" oder "glatt" ausgesagt werden, ist der Gegenstand der Aussage ein individuelles Objekt lediglich als solches. Wo aber Existenz ausgesagt wird, wird von dem individuellen Objekt behauptet, dass es in einer universalen Wirklichkeitssphäre angetroffen wird. "Existieren“ (als Prädikat gebraucht) heisst: seine Stelle in einem universalen Z usammenh ange haben, dessen kategorialer Charakter über ein aus dem Sinn des Urteils erhellendes Minimum hinausliegt. Wenn von dem euklidischen Raume gesagt wird, dass er drei Dimensionen hat, und dass sein Krümmungsmass $=0$ ist, so handelt es sich um Prädikate, die diesen Raum lediglich als für sich vorgestelltes System betreffen; wenn aber von inm gesagt wird, dass er existiert, so heisst dies, dass er Realitäten yon nicht 
bloss mathematischer Art einschliesst: nicht das gesonderte Dasein dieses Raumes ist mehr in Frage, sondern seine Bedeutung für die Welt der kategorial bestimmten Objekte. - In der Schrift über den „Einzig möglichen Beweisgrund zu einer Demonstration des Daseins Gottes" hat Kant die zwar nicht ganz einwandfreie, ${ }^{1}$ ) jedoch gar nicht unbedeutende Formulierung geprägt: „Es ist aber das Dasein in den Fällen, da es im gemeinen Redegebrauch als ein Prälikat vorkommt, nicht sowohl ein Prädikat von dem Dinge selbst, als vielmehr von dem Gedanken, den man davon hat" (S. W., brsg. v. Hartenstein 1867/68, II, 116). Wer z. B. die Ebenheit des Auschauungsraumes bezweifelt, denkt sich den Raum mit nicht-euklidischen Eigenschaften. Ob man nun diesen nichtenklidischen Raum als existiereude und alles Räumliche umfassende Realität vorstellt, oder bloss als Gebilde mathematischer Konstruktion, das ändert nichts an seinen Prädikaten. Aber, würde Kant sagen, an dem Gedanken, den man von diesem nicht-euklidischen Raum hat, ändert es etwas, - und die Meinung, die dieser etwas vage Ausdruck anstrebt, kaun nur die sein, dass der Gedanke von einem Dinge erst dann in Ordnung ist, wenn er die Isoliertheit r'os für sich vorgestellten Dinges überwunden hat. Die Unterscheidung zwischen dem „Ding“ und dem "Gedanken, den man davon hat," wäre also zu ersetzen durch diejenige zwischen dem isoliert aufgefassten und dem im Zusammenhange seiner Wirklichkeits sphäre gedachten Objekte. -

In den bis hierher geführten Erörterungen kam die Eigenart der mathematischen Existenz noch nicht zu ihrem Rechte. Zwar waren die Beispiele fast sämtlich aus dem Gebiete der Mathematik genommen; aber es wäre ebensogut möglich gewesen, dieses Gebiet völlig zu vermeiden und doch dieselben logischen Einsichten zu entwickeln. Soll der besondere Charakter der mathematischen Existenz bezeichnet werden, so ist zunächst zu sagen, dass diese Existenz niemals konkret sondern stets nur abstrakt ist. M. a. W.: mathematische Gegenstände existieren lediglich als Gebilde des reflektierenden Denkens.

Mit dieser Formulierung soll nun Kants Betonung des anschaulichen Charakters der mathematischen Objekte nicht 1880, $11 \mathrm{f}$

1) VgL z. B. Julius Bergmann, Sein und Erkennen, Berlin 
abgelehnt sein: $\left.{ }^{1}\right)$ aber dies ist freilich zu sagen, dass Kant den aktiven Charakter dessen, was in transzendentalen Idealismus Anschauung heisst, zu wenig hat hervortreten lassen, sodass ihm infolgedessen z. B. der Raum, in dem wir uns „vermittelst des äusseren Sinnes (einer Eigenschaft unseres Gemütes) Gegenstände ausser uns vorstellen" (Kr. d.r. V. ${ }^{2}$ 37), und der Raum, der ein Prinzip der Erkenntnis ist, sich nicht hinlänglich gegeneinander abheben. Riehl hat mit grösstem Erfolge daran gearbeitet, den kritischen Kant von den psychologischen Resten zu befreien, die seiner empirisch-historischen Erscheinung noch anhaften; im Zusammenhange mit dieser Arbeit steht auch die Erklärung, dass der nicht eben glüeklich gewählte Kantische Ausdruck „reine Anschauung“ „eine gedankliche Vorstellung“ bezeichne, durchaus nicht etwa ein Sehen (a. a. 0. 457;58). Erkennen ist nicht Wahrnehmen, nicht blosses „Vorstellen vermittelst des äusseren Sinnes", sondern ès ist Überwindung der Wahrnehmung. Diese Überwindung aber ist ihrem Wesen nach Tätigkeit der Reflexion. In der blossen Wahrnehmung gibt es keinen unendlichen und ins Unendliche teilbaren Raum und keine unendliche und ins Unendliche teilbare Zeit, sondern nur das begrenzte, jedoch nicht als begrenzt begriffene, durch sinnliche Qualitäten bestimmte Jetzt und Hier. Infolge psychischer Gewöhnung pflegen Mensch und Tier dem lediglich Wahrgenommenen hinlänglich Rechnung zu tragen: auch ohne darauf zu reflektieren, dass vor ibnen eine Wand ist, versuchen sie gleichwohl nicht, durch sie hindurchzugehen; die wahrgenommene Grenze des Raumes, in dem sie sich befinden, genügt. Allein diese psychologisch erklärbare Sicherheit der Bewegungen ist keine Erkenntnis. Wo hingegen zwar kein Tier, aber ein Mensch șich darüber klar wird, dass er eine Wand vor sich hat, da liefert ihm diese die Wahrnehmung überwindende Reflexion nicht bloss den Gegenstand Wand (ein substanzielles Ding mit bestimmten Akzidenzien), sondern sie liefert ihm notwendigerweise den Gegenstand im Raum: der Gegenstand nimmt seinen Raum ein, und der Raum reicht weiter als der Gegenstand. Vorher gab es nur das unbegriffene begrenzte Hier; jetzt ist diese Grenze durchbrochen. Die Reflexion ist durch sie hindurchgegangen, und sie ist sich dessen gewiss, dass schlecht-

1) Vgl. hierzu und zum folgenden Richard Honigswald, Zum Streit über die Grundlagen der Mathematik, Heidelberg 1912, $46 \mathrm{ff}$. 
hin keine sinnliche Begrenzung endgültig ist. Der Reflektierende, oder - in Kantischer Terminologie - der den Gegenstand im Raum Anschauende sieht nicht mehr als zuvor, aber er weiss mehr: er hat nicht bloss ein durch Sinnesqualitäten in vertrauter Weise bestimmtes Blickfeld, sondern er weiss die Objekte als Gegenstände im Raum. Das Reflektieren hat die der sinnlichen Wahrnehmung gesetzte Grenze durchbohrt, das Bewusstsein geht durch die Objekte hindurch und umfasst sie als ihren Raum erfüllende. - Dieselben Beziehnngen gelten von der Zeit. Es möge etwa in einiger Entfernung von mir ein Holzfäller arbeiten. Ich sehe die Axt anf den Baumstamm einschmettern und höre eine halbe Sekunde später den Schlag. Für ein lediglich passiv wahrnehmendes Bewusstsein bleiben diese Inhalte unverbunden aussereinander. Die Reflexion aber macht aus dem Vorher und Nachher eine objektive Einheit, die Einheit eines zeiterfüllenden Vorganges: die zwischen der Gesichtswahrnehmung und der Gehörsempfindung verflossene Zeit ist erfüllt durch den kausalen Prozess der Bewegung der Schallwellen. Es liegt durchaus nicht so, dass die Reflexion bloss an dem Verhältnis der Wahrnehmungsinbalte zueinander etwas änderte: sie ändert notwendigerweise zugleich etwas an der Zeit - genauer: nur durch sie und nur für sie gibt es diese Zeit, in der etwas zu geschehen vermag. Für das reflexionslose Bewusstsein existiert nur erst die stets sich ändernde und nie als solche begriffene Gegenwart. Die Reflexion allein viermag diese unbegriffene Grenze zu durchdringen und als transzendentale Gesetzmässigkeit zugleich die Zeit und das Geschehen in ihr zu gestalten. Als transzendentale Reflexionsform der Kansalität setzt sich das erkennende Bewusstsein durch die unbegriffene Gegenwartsgrenze hindurch, hebt diese Grenze auf und erweist sich als Bewusstsein der Zeiterfüllung. Das Bewusstsein der Zeit und das Bewusstsein des zeiterfüllenden Vorgangs sind in der Wirklichkeit untrennbar voneinander; nur die Abstraktion vermag hier za scheiden.

Die transzendentale Handlung, die die Begrenztheit des Hier aufhebt und den Raum konstituiert, ist derjenigen, die die Begrenztheit des Jetzt überwindet und die Zeit möglich macht, gleichartig. Das ist freilich eine abstrakte Betrachtungsweise: denn in concreto ist die erstgenannte transzendentale Handlung identisch mit der Kategorie der Raumerfüllung oder Substanzialität, die zweite aber mit der der Kausalität. Allein weil beide Kate- 
gorien sich als Überwindungen unbegriffener Begrenzungen des unmittelbaren sinnlichen Bewusstseins auswirken, erlauben sie diese abstrakte, gleichmachende Betrachtungsweise. Hier wie dort handelt es sich darum, dass sich das erkennende Ich durch das unbegriffene "Nicht-Ich" nicht absolut begrenzen lässt: es setzt sich durch die Begrenzung hindurch; die Selbstgewissheit hört nicht da auf, wo die Wand mein Blickfeld abschneidet, oder wo zusammenhangslose Sinneseindrücke erscheinen und verschwinden. In den Reflexionsbegriffen beherrscht das erkennende Bewusstsein den Raum wie die Zeit. Es dringt prinzipiell durch jede Begrenzung hindurch (wo solche Durchdringung ihm beute nicht gelingt, erkennt es doch eine Aufgabe für morgen). Dieses selbstgewisse SichHineinbohren. in die als Jetzt und als Hier gesetzten Schranken hat Fichte treffend als Linienziehen charakterisiert. Er sagt einmal: „Das sich selbst als tätig anschauende Ich schaut seine Tätigkeit an, als ein Linienziehen. Dieses ist das ursprüngliche Schema der Tätigkeit überhaupt, wie jeder, der jene höchste Anschauung in sich erregen will, finden wird. Diese ursprüngliche Linie ist die reine Ausdehnung, das Gemeinsame der Zeit und des Raumes, aus welcher die letzteren erst durch Unterscheidung und weitere Bestimmung entstehen. Sie setzt nicht den Raum voraus, sondern der Raum setzt sie voraus; und die Linien im Raume, d. h. die Grenzen der in inm Ausgedehnten sind etwas ganz anderes" (S. W. III, 58). Wozu noch daran erinnert werden darf, dass die ursprüngliche Linie für Fichte die gerade ist (vgl. z. B. S. W. I, 64). Kants geniale Thesen über Raum und Zeit siud in diesen Sätzen in sehr erheblicher Weise weiterentwickelt. Und man wird sagen dürfen, dass Kants eigene Auffassung der transzendentalen Ästhetik eine Tendenz zu dieser Fortbildung in sich barg. Neun Jahre nach dem Erscheinen der Kritik der reinen Vernunft spricht Kant davon, dass Raum und Zeit erst dadurch ihre Existenz gewinnen, dass das Erkenntnisvermögen sie aus sich selbst a priori zustande bringt. ${ }^{1}$ )

1) Vgl. S. W., hrsg. v. Hartenstein 1867 f., VI, 37/3.3 [gekürzt]: „Die Kritik erlaubt keine angebornen Vorstellungen; alle insgesamt nimmt sie als erworben an. Es gibt aber auch eine ursprüngliche Erwerbung, folglich auch dessen, was vorher gar noch nicht existiert, mithin keiner Sache vor dieser Handlung angehört hat. Dergleichen ist die Form der Dinge im Raum und der Zeit; unser Erkenntnisvermögen bringt sie aus sich selbst a priori zustande." In solchen Worten ist eine Differenz von der 
Oben wurde gesagt, dass die mathematischen Gegenstände Existenz haben lediglich als Gebilde des reflektierenden Denkens: die jener These folgenden Darlegungen haben entwickein sollen, welcher Art das Material ist, aus dem das reflektierende Denken die mathematischen Gebilde gestaltet: „das Gemeinsame der Zeit und des Raumes", "die reine Ausdehnung" - nicht etwas irgendwie Wahrgenommenes oder "Gegebenes" also, sondern ein reines Produkt der Betätigung des reflektierenden, die sinnliche Begrenztheit überwindenden Bewusstseins. Alle konkrete Existenz (in Natur und Geschichte) ist die synthetische Einheit eines Mannigfaltigen. Mathematische Objekte aber haben nur abstrakte Existenz (sie existieren nur als reine Produkte der Reflexion): synthetische Einheiten eines Mannigfaltigen aber sind auch sie. ${ }^{1}$ ) Die Zahl 3 z. B. fasst die Mannigfaltigkeit von drei Einheiten zu einer neuen Einheit zusammen; alles Zählen setzt Mannigfaltiges voraus, das durch das Zählen zu einer Einheit zusammengefügt ist. Die Formulierung "synthetische Einbeit des Mannigfaltigen" gibt also noch nicht den Unterschied zwischen konkreter und abstrakter Existenz an; aber eben dadurch, dass sie ibn nicht angibt, treibt sie anf ihn hin: denn es kann nicht entgehen, dass "synthetische Einheit des Mannigfaltigen" etwas wesentlich anderes meint, wenn z. B. die synthetische Einheit der Eigenschaften weiss, hart und süss in dem konkret existierenden Zucker gedaclit wird, als wenn die synthetische Einheit von 1 und 1 und 1 in der nur durch und für die Reflexion existierenden Zahl $3 \mathrm{zu}$ denken ist. Im ersten Fall sind die in der synthetischen Einheit gedachten Elemente voneinander unterschieden, im zweiten Falle sind sie einander gleich, oder vielmehr: sie sind als gleich behandelt, weil von aller Verschiedenheit abstrahiert ist. In diesem Sinne formuliert Hegel, das Element der Zahl sei „der gleichgültig gewordene Unterschied“, sie habe es "nicht mit Entgegengesetztem zu tun", sie gehe "nur an dem Faden ihrer eigenen Identität fort". Alles Zählen setzt zwar Verschiedenheit voraus, ist aber selbst deren Unterdrückung; wäre zwischen zwei Häusern überhaupt keinerlei Unterschied, so wären es gar nicht zwei Häuser, sondern nur die Identität eines und desselben Hauses. Andrerseits aber sind zwei

Lehre Fichtes überhaupt nicht $z u$ bemerken - wenigstens wenn man mehr als blosse Psychologie in ihnen sucht.

') Vgl. Riehl, a. a. 0. 443. 
Häuser zwei doch nur, sofern die Besonderheiten eines jeden nicht in Frage kommen: sofern sie in ihrer Unterschiedenheit gedacht werden, sind sie nicht addierbar; addierbar ist nur das Gleiche. Selbst das Königreich Serbien, das Kausalprinzip und eine Nikotinvergiftung werden dann zueinander addierbar und machen zusammen drei aus, wenn von ihrer Unterschiedenheit abstrahiert wird und sie etwa lediglich als drei Denkinhalte angesehen werden. Dies also entscheidet: konkrete Existenz bewahrt die Besonderheiten der zur Einheit verschmolzenen Mannigfaltigen; alle Kategorien des empirischen Denkens sind Gestalten solcher wesenhaften Einheiten des Mannigfaltigen. Die mathematische Existenz aber hat ibren "Stoff" lediglich in den reinen Produkten der Reflexionstätigkeit selbst. Eine Beziehung auf die konkrete Wirklichkeit kann (durch Zählen und Messen) nur in der Art stattfinden, dass alle Besonderheit zurückgelassen wird: aufgenommen wird nur dasjenige, was in die Sphäre der „reinen Ausdehnung“ fällt, dasjenige also, was das Ding zum raum- und zeiterfüllenden macht, und was seinen Ursprung in der Tätigkeit hat, durch die das reflektierende Subjekt sich ein Bewusstsein des Dinges konstruiert hat durch Überwindung der sinnlichen Gebundenheit. Wenn etwa gesagt wird, eine bestimmte Stadt babe 60000 Einwohner, so existiert die Zahl 60000 weder ausser, noch über, noch in den wirklichen Einwohnern. Diese existieren als konkrete, von einander verschiedene Menschen: allein hiervon redet jene Aussage nicht. Die Verschiedenheit der Einwohner wird nur vorausgesetzt, nicht ausgesagt. Die statistische Aussage behauptet bloss, dass das abstrakte Einwohnerquantum durch die Summe (die synthetische Einheit) von 60000 Einheiten bezeichnet wird. Aber diese Summe, in der die Einwohner bloss als Einheiten und mithin als gleichartig behandelt sind, existiert ausschliesslich als Gebilde der Reflexion: nur in ihr machen jene 60000 sehr verschiedenen konkreten Menschen eine synthetische Einheit ohne innere Unterschiede aus. Zahlen existieren lediglich in abstracto. - Allein wie verhält es sich mit dem ebenen oder mit dem sphärischen Dreieck? Ist nicht der Übergang von dem Begriff des Dreiecks zu dem des ebenen oder dem des sphärischen Dreiecks eiu Fortschreiten aus dem Abstrakteren ins Konkretere? Und wenn noch die Länge der Seiten und die Grösse der Winkel bestimmt wird wird dann nicht das Denken dieses mathematischen Objektes immer konkreter? In keiner Weise. Nicht der Weg zur.Konkret- 
heit, sondern der zu grösserer Bestimmtheit ist hier bezeichnet: aber diese Bestimmtheit ist durchaus nur eine solche der Reflexion. Zugrunde liegt immer nur die reine Ausdehnung, die ihre Modifikationen entwickelt. Allerdings handelt es sich beim ebenen oder sphärischen Dreieck (anders als bei den ganzen Zahlen) um eine Synthesis verschieden gestalteter Inhalte: aber die Inhalte sind wesensgleich, und infolgedessen verlieren sie in der Synthesis ihre Unterschiedenheit. Die weisse Farbe eines konkret existierenden Stückes Zucker ist als Farbe dieses bestimmten Objekts wahrnehmbar, vorstellbar, denkbar, ohne gleichzeitiges Vorstellen seiner Härte und seines Geschmacks; diese anderen Qualitäten tragen nicht das mindeste zur Vorstellbarkeit der Farbe bei: die einzelnen Eigenschaften haben in der Synthesis ihre Selbständigkeit. Aber die Gleichseitigkeit eines bestimmten gleichseitigen ebenen Dreiecks lässt sich durchaus nicht vorstellen ohne die übrigen Eigenschaften, die den Begriff dieser Figur bestimmen. Die Gleichseitigkeit eines ebenen Dreiecks ist anders vorzustellen als die eines sphärischen: die Eigenschaften des rein mathematischen Objekts gehen infolge ihrer gemeinsamen Herkunft aus der reinen Ausdehnung ineinander auf und büssen jede Selbständigkeit gegeneinander ein. Gerade die Selbständigkeit aber, mit der sich die einzelnen Eigenschaften trotz aller sie umschliessenden Synthesen gegeneinander halten, ist das Charakteristische der konkreten Existenz.

In den Prolegomenen Kants heisst es ( 110$)$ : „Geometrie legt die reine Anschauung des Raumes zum Grunde. Arithmetik bringt selbst ihre Zahlbegriffe durch sukzessive Hinzusetzung der Einheiten in der Zeit zustande“. Dass aber die Beziehung der Arithmetik zur Zeit von sehr viel fernerer Art ist als die der Geometrie zum Raum, spürt jeder aufmerksame Leser, und Kant selbst hat den Unterschied empfunden, wie die in seinem Text unmittelbar folgenden Worte verraten. In IVahrheit hat die Arithmetik mit dem Raum ebensoviel zu tun wie mit der Zeit. ${ }^{1}$ ) Das Element der Zahlenreihe ist "die reine Ausdehnung, das Gemeinsame der Zeit und des Raumes, aus welcher die letzteren erst durch Unterscheidung und weitere Bestimmung entstehen" (vgl.

1) Vgl. Riehl a.a. O. 535: „Die Zahl bezieht sich a priori ebensowohl auf die ruhende Mannigfaltigkeit im Raume wie auf die sukzessiv entwickelte Reihe in der Zeit. Es ist von grösster Wichtigkeit, dass die Zahl dem Raume und der Zeit gemeinsam ist. Die Zaahl ist die logisch bestimmte Mannigfaltigkeit überhaupt." 
oben S. 8). ${ }^{1}$ ) Eine Zahl ist etwas Abstrakteres als ein Gegenstand von zeitlicher oder räumlicher Ausdehnung, und die Arithmetik ist mithin abstrakter als die elementare Geometrie. Berücksichtigt man indessen die Möglichkeit, räumliche Ordnungssysteme auch mit anderen als den euklidischen Eigenschaften zu konstruieren, so erkennt man die Unabhängigkeit der als Prinzip der Konstruktion dienenden "reinen Ausdehnung“ von den Besonderheiten des dreidimensionalen euklidischen Raumes, der seine bevorzugte Stellung einer empirischen Tatsache verdankt und für die mathematische Betrachtung keinerlei Auszeichnung vor einem vier- oder fünfdimensionalen Raume besitzt: denn für die mathematische Betrachtung kommt seine Bedeutung für die konkrete Wirklichkeit nicht in Frage, sondern hier existiert er lediglich als Gebilde einer willkürlichen Konstruktion.

Von der „reinen Ausdehnung“, die soeben als Prinzip nichteuklidischer Geometrien bezeichnet wurde, ist, wie mehrfach erwähnt, die Geradlinigkeit unabtrenubar. Ein "Raum" (von n Dimensionen), in dem die Gerade durch die Geradeste ersetzt wird, hat als Gebilde mathematischer Konstruktion niemals Selbstständigkeit, sondern er lässt sich nur begreifen, indem man ihn hineinkonstruiert in einen ebenen Raum (von $n+1$ Dimensionen). Das übliche Beispiel der zweidimensionalen Kugeloberfläche, auf der eine nicht-euklidische Geometrie gilt, wird mathematisch begreiflich nur, indem die Kugel als stereometrisches Gebilde in den dreidimensionalen ebenen Raum gesetzt wird. Man kann die

1) Rickert leitet in seinem tief dringenden Aufsatz „Das Eine, die Einheit und die Eins" (Logos II, Heft 1) die Zahlen aus den "homogenen Medien" Raum und Zeit her. "Die nicht weiter zu definierende, sondern nur durch Hinweis auf Raum und Zeit verständlich zu machende Homogeneität des Mediums" führt hinaus über die „Besonderheiten des Raumes und der Zeit". Also auch Rickert erkennt, wie Fichte, ein Gemeinsames der Zeit und des Raumes. Doch genügt ihm das „homogene Medium“ noch nicht. Es gewährt noch keine Möglichkeit der Ordnung: „Die Gegenstände. bilden ein regelloses, verwirrendes Gewühl" (62). Diesem Mangel wehrt der noch hinzutretende Begriff der „Reihe“ (67). Solange man von Raum und Zeit als nun einmal vorhandenen Tatsachen ausgeht, ist diese Ergänzung des ihnen Gemeinsamen durch den Begriff der Reihe völlig berechtigt und notwendig. Für Fichte stellt sich das Problem anders. Er kommt nicht von Raum und Zeit her zu der ihnen gemeinsamen "reinen Ausdehnung", sondern von dieser aus konstruiert er jene; in der "reinen Ausdehnung" aber hat er bereits die Geradlinigkeit und damit das ordnungbegründende Prinzip. 
ouklidische Geometrie als einen Spezialfall verschiedener möglichen Geometrien ansehen, aber man muss der "Hypothese des rechten Winkels" zugestehen, dass sie als Norm alles geometrischen Verstehens unentbehrlich ist. Unter diesem Gesichtspunkt ist zu der Frage, ob die auf das Parallelenaxiom gebaute Geometrie von unserm Erfahrungsraum gelte, zu bemerken, dass sie ohne alleu Zweifel bejaht werden muss: nur dies darf gefragt werden, ob ein System von drei Dimeusionen genügen wird, um die in der Erfahrung sich ergebenden räumlichen Beziehungen vollkommen zu bestimmen. Die mathematische Reflexion kann die gerade Linie niemals los werden: denn uur von ihr hat das erkennende Subjekt ein unmittelbares Selbstbewusstsein, während eine "Geradeste“, um in ihrer mathematischen Eigenart begriffen zu werden, einer das Gesetz ihrer besonderen Krümmung ausdrückenden Formel bedarf, also nur mittelbar gewusst werden kann. ${ }^{1}$ ) Darum ist uur die Gerade eindeutig; unser Wissen von ibr ist etwas ganz anderes als die blosse Feststellung einer Invariante; eine solche hat keine Selbstgewissheit. Würde sich eine Geometrie auf eine solche aufbauen, so wäre ihr letztes Element unbegriffen. Nicht von einer kürzesten Linie überhaupt, sondern nur von der absolut kürzesten wissen wir, was sie ist: jede nur relativ kürzeste ist unbegriffen, solange die Relation unbekannt oder gar ungeahnt bleibt, in der sie zur Geraden steht. In der Geraden schaut, mit Fichte zu reden, das Ich seine eigene Tätigkeit an; sie ist die reine Selbstdarstellung des Ich, das sich seiner sinulichen Schranken bewusst wird, indem es sie durchdringt, - Dieses Ich also, das selbst nur erst abstrakte Wirklichkeit hat, sofern die Realität noch ein unbuzwungenes Nicht-Ich ihm gegenüber ist; das aber der Notwendiogkeit gewiss ist, sich in das Nicht-I'h hineinzuwerfen, um es sich anzueignen; und das sich dessen gewiss ist, dass es sich im chaotischen Strudel des Nicht-Ich nicht verlieren wird: dieses völlig inhaltlose und dabei doch völlig selbstgewisse Ich schafft aus sich selbst die mathematischen Gebilde, denen allen es sein eigenes Wesen mitgibt: seine Inhaltlosigkeit und seine selbstgewisse Notwendigkeit und seinen kühnen Mut, es mit der konkreten Welt aufzunehmen. -

1) Vgl. hierzu Bruno Bauchs Ausführungen über die euklidische Geometrie als die einzige "Möglichkeitsgrundlage für Wirklichkeitserkenntnis" (Studien zur Philosophie der exakten Wissenschaften, Heidelberg 1911, $127 \mathrm{ff}$.). 
Dass alle mathematische Existenz abstrakt ist, d. h. nur durch und für das reflektierende Denken besteht, ist dargelegt. Aus dieser Einsicht aber entspringt. eine Frage: In der von allgemein logischen Erwägungen ansgehenden Einleitung dieser vorliegenden Abhandlung ist gezeigt worden, dass jede Existenzaussage die Zugehörigkeit zu einem Existenzzusammenhang von bestimntem Grade zu ihrem Inhalt hat, und zwar von einem höheren Grade als dem als selbstverständlich vorauszusetzenden. Welches ist nun in dem Falle der Mathematik der als selbstverständlich vorauszusetzende Existenzgrad, über den die rein abstrakte Existenz der Konstruktionsgebilde sich als höherer Grad erhebt? Die Antwort muss sich finden lassen, wenn man davon ausgeht, dass, wie die einleitenden Ausführungen klargelegt Laben, das negative Existenzialurteil dem Urteilssubjekt niemals jedwede Existenz abspricht, sondern nur dies besagt, dass die Existenz des Urteilssubjekts die als selbstverständlich vorausgesetzte Sphäre nicht überschreitet. "Es gibt kein gleichseitiges rechtwinkliges Dreieck": hier ist - so wird behauptet - dem gleichseitigen rechtwinkligen Dreieck nicht alle Existenz abgesprochen; und die Sphäre, in der es unangefochten existiert, ist diejenige, die in jedem .mathematischen Existenzialurteil vorausgesetzt ist. Aber welches ist diese Sphäre?

Offenbar versteht man einen negativen Existenzialsatz nur dann, wenn man sich zunächst unter seinen Elementen etwas denken kann. Wer nicht weiss, was ein Häufungswert ist, hat keine Meinung über die Richtigkeit des Urteils: „Es gibt keinen Häufungswert der Zahlenfolge 1, 2, $3 \ldots$. ; der aber, dem die hier in Frage kommenden Begriffe geläufig sind, vermag die Richtigkeit des negativen Urteils daran zu erkennen, dass er die Unmöghichkeit der dem konstruierenden 'Denken gestellten $\mathrm{Zu}$ mutung einsieht. Und ebenso gründet sich die Ablehnung des gleichseitigen rechtwinkligen Dreiecks auf die Einsicht des reflektierenden Subjekts, dass in dieser Wortverbindung Unmögliches von ihm verlangt wird. Allgemein gesprochen: Der Gegenstand eines mathematischen Existenzialsatzes ist in jedem Falle zu verstehen als die Formulierung einer Aufgabe für das konstruierende Denken; das affirmative Urteil spricht die Lösbarkeit, das negative die Unlösbarkeit der Aufgabe aus. Durch die Lösung findet das nunmehr konstruierte Objekt seine positive Stelle in den Zusammenhängen des mathematischen Denkens. Das Nicht- 
Konstruierbare aber hat keine Existenz im Sinne der Mathematik: es hat lediglich die Existenz einer mathematischen Aufgabe, einer Aufgabe für jenes allerabstrakteste Reflektieren, das den jeder Konkretheit entkleideten Gegenstand, die "reine Ausdehnung“ zum Material hat. Nunmebr wird deutlich, welches die beiden im mathematischen Existeuzialurteil in Betracht kommenden Existenzsphären sind: Die im affirmativen Urteil bebauptete Lösbarkeit einer mathematischen Aufgabe ist die Statuierung irgendwelcher bestimmten Quantitätsbeziehung; ihrer Möglichkeit aber geht voraus die noch ungelöste - und vielleicht unlösbare - Aufgabe, die nun in die Sphäre der noch unbestimmten Grössenbeziehungen fällt. Die den wesentlichen Charakter des Aufgegebenen bezeichnenden Eigenschaften zwar werden auch hier bestimmt gedacht; anderenfalls hätte die Aufgabe selbst keine Bestimmtheit: unbestimmt und problematisch aber bleibt die Einheit jenes Mannigfaltigen, d. h. die Möglichkeit, jene Eigenschaften in der Konstruktion eines neuen Gebildes derart zu verschmelzen, dass sie alle Selbständigkeit gegeneinander verlieren. Man denke an das gleichseitige rechtwinklige Dreieck; oder, wenn man ein sinnvolleres Beispiel will, an die irrationalen Zahlen: Dass es Verhältnisse linearer Grössen gibt, die durch keine rationale Zahl auszudrücken sind, war schon den alten Griechen bekannt; arithmetische Definitionen der irrationalen Zahlen aber sind erst in der zweiten Hälfte des 19. Jahrhunderts (durch Dedekind, Weierstrass, Georg Cantor) geleistet worden. Die wesentlichen Eigenschaften also, die diese Zahlen haben sollten, waren in voller Bestimmtheit gedacht: ein Problem war nur die Hauptsache, ob nämlich jene (bei linearen Grössen festgestellten) Eigenschaften auch die Eigenschaften von Zahlen sein könnten, ob, mit einem Worte, irrationale Zahlen existierten. Bevor sie auf arithmetische Weise definiert waren, existierten die irrationalen Záhlen lediglich als ungelöste Aufgaben in der Sphäre der noch unbestimmten Quantitätsbeziehungen - ebenso wie das gleichseitige rechtwinklige Dreieck, von dem freilich feststeht, dass es nie zur Bestimmtheit einer gegenständlichen Einheit gelangen kann. - Die „reine Ausdehnung“ ist sichtlich anch für diese niedrigste Existenzśphäre der „Stoff“, und da schon dieser ein Erzeugnis der Reflexion ist, so ist auch die Existenz der ungelösten mathematischen Aufgabe die abstrakte Existenz eines - nun freilich unbestimmten - Reflexionsgebildes. Sie unterscheidet sich von der Existenz des bestimmten mathe- 
matischen Objektes dadurch, dass das die synthetische Einheit des. Mannigfaltigen gestaltende Prinzip - auch das ungelöste Problem ist nicht blosse Mannigfaltigkeit, sondern eine Einheit des Mannigfaltigen! - im Falle des konstruierten mathematischen Objektes die oben geschilderte Verschmelzung, das Ineinander-Aufgehen der Eigenschaften ist, im Falle der blossen mathematischen Aufgabe aber die Forderung, das Sein-Sollen einer solchen VerschmelzungAls Problem existieren heisst: wie die einander suchenden Hälften

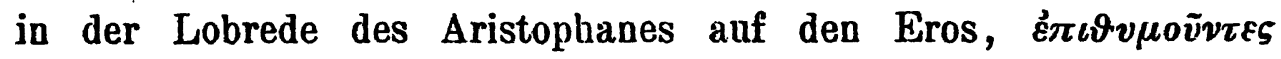
$\sigma u \mu \varphi \tilde{v} \nu \alpha$, in einem Bewusstsein der begehrten Einheit vereint sein. Die Einheit der Mannigfaltigen ist keine vollzogene (bestimmte), sondern eine angestrebte (unbestimmte); die "Gegenständlichkeit" des Problems als solchen ist begründet durch das Bewusstsein einer sein-sollenden Verschmelzung der Mannigfaltigen. Die Verschmelzung ist eine bloss sein sollende, nicht eine wirkliche: d. h. es ist unbestimmt, wie sie geschehen kann; sowie diese Frage in positivem Sinne entschieden ist, ist die höhere Existenzsphäre der bestimmten Quantitätsbeziehungen erreicht und aus der mathematischen Aufgabe ein mathematisches Objekt geworden. -

Die für das logische Verständnis des mathematischen Existenzialurteils entscheidenden Gesichtspunkte sind hiernit anfgewiesen. Aber eine bestimmte Klasse von Urteilen, die ganz besondere Schwierigkeiten zu machen scheint, soll noch erwähnt werden. Wie steht es mit Urteilen, in denen die Existenz zahlenmässig bestimmt ist? „Es gibt 2 Häufungsstellen der Folge $\frac{1}{3}, \frac{2}{3}, \frac{1}{4}, \frac{3}{4}, \frac{1}{5}, \frac{4}{5} \ldots$.. Geht man davon aus, dass der mathematische Existenzialsatz die Lösbarkeit einer Aufgabe behauptet, so leuchtet sofort ein, dass solche Urteile eine besondere Stellung einnehmen. In den bisher betrachteten Existenzialsätzen war der Inhalt der Aufgabe, das mathematische Objekt, dessen Existenz behauptet werden sollte, zu konstruieren. Niemand wird aber behaupten wollen, dass das soeben angeführte Beispiel als Lösung folgender Aufgabe anzusehen sei: „Es sind die beiden Häufungsstellen der Folge $\frac{1}{3}, \frac{2}{3}, \frac{1}{4}, \frac{3}{4}, \frac{1}{5}, \frac{4}{5} \ldots$ zu konstruieren". Sondern die ihm vorauszusetzende Aufgabe kann nur gewesen sein: "Wie viele Häufungsstellen hat die Folge $\frac{1}{3}, \frac{2}{3}, \frac{1}{4}, \frac{3}{4}, \frac{1}{5}, \frac{4}{5} \ldots$ ?" Wird man nun aber sagen wollen, die Antwort auf diese Frage sei ein Existenzialurteil? 'Mit gleichem Rechte müsste man die Antwort auf die Frage "Wie viel Saiten hat die Gitarre?" als Existenzialurteil gelten lassen. Doch der entscheidende Grund, in dem frag- 
lichen Urteil lediglich eine Eigenschaftsaussage über die Folge $\frac{1}{3}, \frac{2}{3}, \frac{1}{4}, \frac{3}{4}, \frac{1}{5}, \frac{4}{5} \ldots$. aber keinen Existenzialsatz anzuerkennen, liegt. in folgendem: Wie in den einleitenden Darlegungen dieses Aufsatzes gezeigt worden ist, ist es das Wesen des Existenzialsatzes, das Verhältnis eines Objektes zu. einem universalen Wirklichkeitszusammenhang festzulegen. Wo Existenz ausgesagt wird, wird ein Objekt unmittelbar von einer transzendentalen Kategorie beansprucht, und jede solche Kategorie bedeutet die grenzenlose Wirklichkeit. Wo immer aber es sich um zahlenmässig bestimmte Existenz handelt, macht bereits die natürliche Betonung beim Aussprechen deutlich, dass es sich um die zahlenmässige Bestimmung handelt, nicht aber um die Existenz (die hier nur ebenso vorausgesetzt wird, wie die Existenz der Gitarre bei der Angabe ihrer Saitenzahl). Folglich aber ist keine Rede davon, dass der Gegenstand eines solchen Urteils unmittelbar in den Totalitätszusammenhang einer Kategorie eingestellt würde. In dem angeführten Beispiel werden die beiden Häufungsstellen vielmehr ganz bescheidentlich zunächst in den minimalen Zusammenhang einer beschränkten Zahlenfolge hineingedacht; dass dann weiterhin diese Zahlenfolge in der Totalität der mathematischen Realitäten ihre Stelle hat, ist zwar richtig, steht aber hier durchaus nicht in Frage. -

Zum Schluss ein Wort über das Verhältnis von Möglich keit und Existenz in der Mathematik. In älterer, vorkantischer, wie in neuerer, von Kant beeinflusster Zeit - die Kritik der reinen Vernunft bedeutet dieser Frage gegenüber zum mindesten eine enorme Erweiterung des Gesichtswinkels - ist die mathematische Existenz oft der Möglichkeit schlechthin gleichgesetzt worden; so in jüngster Zeit z. B. von Hugo Bergmann. ${ }^{1}$ ) Hier sei zunächst darauf hingewiesen, dass in den vorstehenden Erörterungen die Existenz zwar mit der „Möglichkeit der Konstruktion“, aber nirgends mit der Möglichkeit ohne weiteres identifiziert worden ist. Mathematische Existenz hat nur dasjenige, was konstruiert werden, d. h. was als bestimmtes Gebilde aus dem Material der reinen Ausdehnung geschaffen werden kann, die ihrerseits ihren Ursprung in der Überwindung des unbegriffenen Jetzt und Hier hat. Die Konstruktion gibt dem in mathematischer Reflexions-

1) Über den analytischen Charakter des Existenztheorems in der reinen Mathematik, Ostwalds Annalen der Naturphilosophie, Bd. VIII, $495 \mathrm{ff}$. 
weise Gedachten seine bestimmte Gegenständlichkeit, sie ist der Beweis seiner Existenz. Aber kann man sagen, dass z. B. eine imaginäre Zahl konstruierbar sei und demzufolge existiere? $\sqrt{-1}$ bedeutet eine unvollziehbare Aufgabe; das reflektierende Denken kann den Inhalt dieses Symbols nicht bestimmen; $n^{\mathrm{i}}{ }^{\text {“ }}$ ist nicht konstruierbar, es existiert nicht. . Und dasselbe muss von allen Erzeugnissen mathematischer Kunstgriffe gesagt werden. In der Mathematik, sagt Frege ${ }^{1}$ ) ist „ein Begriff zulässig, auch wenn seine Merkmale einen Widerspruch enthalten; man darf nur nicht voraussetzen, dass etwas unter ihn falle". So ist "i" allerdings in der Mathematik zulässig, d. h. möglich: da aber niemals „etwas“, d. h. etwas Ausgedehntes $=i$ sein kann, hat es keine Gegenständlichkeit, keine Existenz im Sinne der Mathematik. Es ist kein bestimmtes Gebilde des reflektierenden und konstruierenden Denkens, soṇdern bloss eine Aufgabe für dieses: Existenz hat es nur im Limbus der unerlösten Probleme. Diese Existenz ist mathematisch wertlos: sein mathematischer Wert besteht in seiner Möglichkeit, d. h. darin, dass man mit ihm operieren kann. Was für sich selbst den Bedingungen der Konstruierbarkeit widerspricht, kann gleichwohl in den Zusammenhängen der auf ein konstruierbares Ziel gerichteten Reflexion seine Stelle erhalten. Dèr Beweis der mathematischen Möglichkeit liegt lediglich in solcher tatsächlichen Brauchbarkeit; er wird also in der Sphäre der mathematischen Existenz geführt. Das mathematisch bloss Mögliche steht somit zwar seinem Existenzcharakter nach auf keiner höheren Stufe als das Phantastische und Unmögliche: es erhebt sich aber, oder vielmehr es wird erhoben zu höherer Bewertung (nicht zu höherer Existenz) um einer Tauglichkeit willen, die. es nicht von sich selbst hat, sondern die es aus der ihm überlegenen Existenzsphäre empfängt; und in diese tritt es ein - nicht zu endgültigem Bestand (denn das wäre Existenz) -, aber um in ihr aufgehoben und einem existenten Zwecke dargebracht $\mathbf{z u}$ : werden.

1) G. Frege, Die Grundlagen der Arithmetik, Breslau 1884, 105. 